\title{
Искусственный интеллект и рентабельность как движущие факторы развития САПР
}

\author{
М. Макушин ${ }^{1}$, А. Фомина, д. э. н. ${ }^{2}$
}

УДК 658.512 | ВАК 05.13.12

\begin{abstract}
Применение инструментальных средств САПР - один из ключевых факторов совершенствования ИС и обеспечения их дальнейшего масштабирования. Именно от них во многом зависит ускорение вывода на рынок новой продукции благодаря сокращению цикла разработки. Динамика продаж САПР и доходов от оказания услуг проектирования ИС в целом коррелируется с динамикой продаж ИС, правда, с небольшим временным разрывом. К основным факторам развития САПР относятся рентабельность и искусственный интеллект.
\end{abstract}

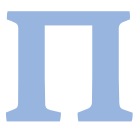

о итогам 2018 года продажи полупроводниковых приборов составили около 430 млрд долл., а в 2023-м могут превысить 570 млрд долл. [1]. Что касается отдаленных перспектив, то прогнозируется расширение этого рынка до 1 трлн долл. в 2025-2027 годах. Обеспечение такого роста невозможно без совершенствования существующих и создания новых инструментальных средств САПР. В целях повышения степени автоматизации САПР и сокращения доли "ручных" работ предполагается шире использовать методы искусственного интеллекта и машинного / глубокого обучения. При разработке продукции поставщики САПР ориентируются на наиболее перспективные изделия микроэлектроники, отличающиеся высоким спросом. Наряду с этим все более широкое развитие получают услуги виртуального проектирования, рынок облачных САПР. Основной проблемой поставщиков САПР становится поставка заказчикам подстроенных под их требования решений, обеспечивающих рентабельность хозяйственной деятельности.

\section{РЫНОК САПР}

По данным исследовательских фирм Mordor Intelligence и Research and Markets, мировой рынок САПР, достигший достигший в 2018 году 9,76 млрд долл., к 2019 году, может увеличиться до 11 млрд долл. в год, а в 2024-м - превысить 17,35 млрд долл. Соответственно, среднегодовые темпы роста продаж в прогнозируемый период (CAGR) составят 10,1\% (рис. 1). Инструментальные средства САПР - один из основных факторов, обеспечивающих инновационный потенциал и рентабельность микроэлектроники. Их развитие

АО «ЦНИИ «Электроника», главный специалист,

mmackushin@gmail.com.

АО «ЦНИи «Электроника», генеральный директор. содействует освоению новых проектных норм, усложнению конструкций, интеграции в них перспективных типов сложнофункциональных (СФ) блоков, ускорению и удешевлению процессов проектирования и вывода на рынок ИС.

растущий спрос на современные электронные приборы со сложными конструкциями и необходимость уменьшения размеров ИС при одновременном повышении их производительности вынуждают производителей наращивать инвестиции в НИОКР и внедрение инструментальных средств САПР. Ожидается, что в ближайшее время данный сектор продемонстрирует одни из самых высоких темпов роста в отрасли.

\section{Основные тенденции рынка САПР}

Во-первых, производители полупроводниковыхприборов прилагают дополнительные усилия к созданию и производству автомобильных ИС, готовясь к существенному

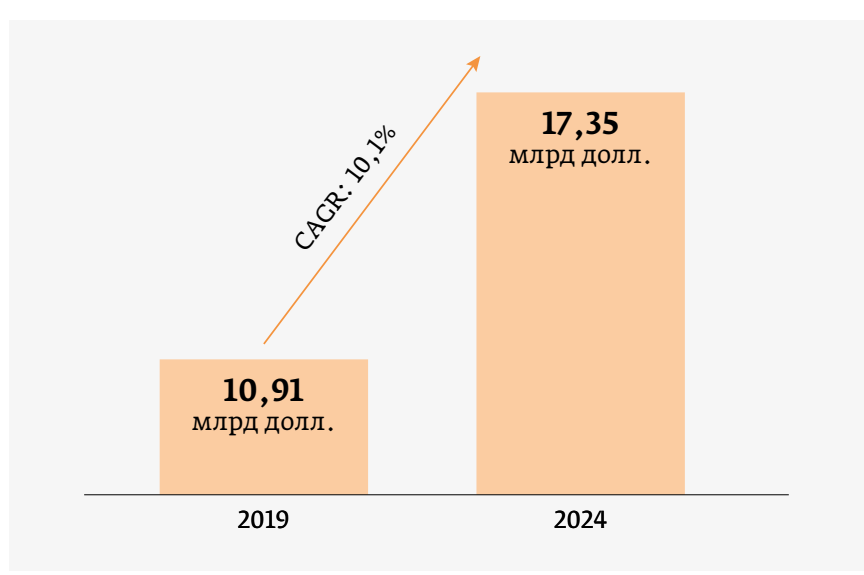

Рис. 1. Прогноз динамики рынка САПР в 2019-2024 годах. Источник: Mordor Intelligence 
росту спроса на изделия, используемые в перспективных системах помощи водителю (ADAS), электромобилях/гибридных машинах, а также в автономных транспортных средствах. Основные поставщики САПР пытаются расширить портфели процессов / СФ-блоков для производителей автомобильной электроники. Автомобильный сектор привлекателен, в частности, тем, что многие используемые ИС не требуют применения передовых процессов (например, ИС Интернета вещей (IOT) для подключаемых к сетям автомобилей / автономных транспортных средств), следовательно, проектировать и производить такие ИС с умеренными издержками может большое число поставщиков.

Во-вторых, неотъемлемой частью производства ИС стали виртуальные проектирование и верификация конструкций - из-за растущих требований к безопасности и производительности. Этот процесс предусматривает тестирование электрических и электронных блоков при различных значениях температуры и давления. Растущая сложность автомобильных, медицинских, промышленных и других систем стимулирует спрос на инструментальные средства САПР. Поэтому поставщики начали предлагать свои инструментальные средства и ПО в Интернете, что позволило потенциальным клиентам получить легкий доступ и использовать эти средства для проектирования ИС. Следовательно, капитальные затраты компаний уменьшились вследствие снижения ТСО и затрат на инфраструктуру для размещения этих инструментальных средств [2, 3].

В-третьих, доступность инструментальных средств САПР в Интернете привела к формированию рынка облачных САПР. Основными факторами роста этого сегмента можно считать преимущества лицензирования (плата только за использование значительно снижает издержки), отсутствие необходимости приобретать специализированные системы и решение проблемы хранения данных (все в облаке). По данным исследовательской фирмы Market Research Future, мировой рынок облачных CAПР за 2016-2022 годы продемонстрирует CAGR порядка 5,51\% (КНР - 6,11\%), а объем доходов к концу прогнозируемого периода составит 7,596 млрд долл. Крупнейшим сегментом в структуре облачных САПР стала разработка и реализация полупроводниковых СФ-блоков - его объем в 2022 году достигнет 2,746 млрд долл. В сфере проектирования конечных электронных систем наибольшую долю займут системы военного назначения - 2,418 млрд долл. в 2022 году, а по уровню CAGR в 2016-2022 годах на первом месте будут телекоммуникационные системы -6,84\%.

\footnotetext{
TCO (Total Cost of Ownership) - общая (совокупная) стоимость владения, ССВ методика определения оптимального соотношения цена / качество для оборудования и ПО. Критериями оценки являются стоимость приобретения, установки, администрирования, технической поддержки и сопровождения, модернизации, вынужденных простоев и других затрат.
}

В течение прогнозируемого периода на рынке облачных САПР будут доминировать США, но их достаточно быстро будет настигать КНР. К основным игрокам в этом сегменте относятся Cadence Design Systems, Mentor Graphics, Synopsys, Agilent, Agnisys, Aldec, Ansys, JEDA Technologies, MunEDA, Sigrity и Zuken [4].

B-четвертых, лучший показатель CAGR в 2018-2023 годах продемонстрируют секторы оказания услуг $(14,1 \%)$ и разработки / реализации полупроводниковых СФ-блоков, которые в 2017 году суммарно занимали 38\% рынка в целом (рис. 2). К данным секторам относятся консультации по установке инструментальных средств САПР, их обслуживанию и обновлению, а также приобретение лицензий и подписок. Конечным пользователям также предоставляются услуги по обучению и поддержке. Рост этого сегмента зависит в основном от спроса на САПР. По объему доходов от этого вида деятельности лидируют американские фирмы, что обусловлено благоприятной государственной политикой и растущим спросом на потребительскую электронику в США, связанным, в частности, с распространением Іот (умные дома, автономные транспортные средства и т.д.) [5].

\section{Географическая структура рынка САПР}

Наибольшая доля рынка САПР и связанных с ними секторов СФ-блоков / услуг приходится на Америку (в основном США). Однако в сегменте установленного полупроводникового оборудования первое место уже около пяти лет удерживает Тайвань, за ним следует Южная Корея, быстро растут рынок и производство полупроводниковых

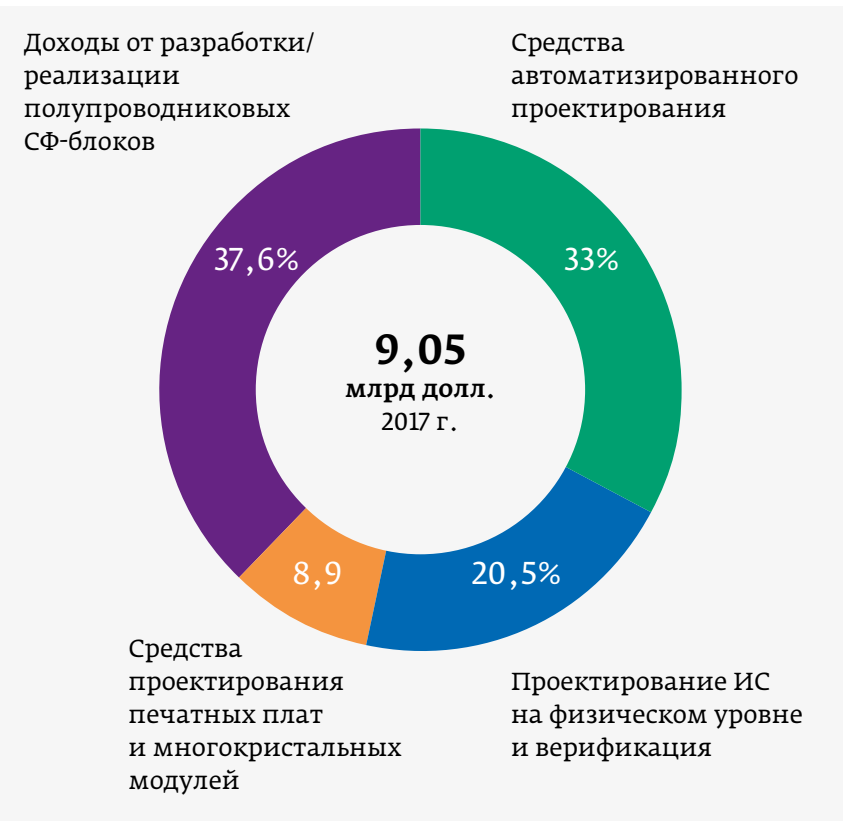

Рис. 2. Структура мирового рынка САПР в 2017 году. Источник: Mordor Intelligence 
приборов в КНР. Все это указывает на то, что в обозримом будущем пальма первенства перейдет к странам Азиатско-Тихоокеанского региона (АТР). Особой тенденцией на китайском рынке последних лет стала консолидация, в рамках которой под контролем государства китайские игроки объединились для наращивания производственных мощностей и повышения конкурентоспособности. Новые структуры могут использовать свои финансовые ресурсы и производственные мощности для увеличения внутреннего производства и снижения зависимости страны от импорта. Соответственно, растет их потребность в САПР. При этом правительство КНР активно поддерживает развитие национальной индустрии САПР [2, 3].

По данным ESD Alliance, в I квартале 2018 года продажи САПР и полупроводниковых СФ-блоков в Америке впервые превысили 1 млрд долл. (табл. 1). Численность занятых в этой сфере в мире увеличилась на 10,4\% - с 37,2 тыс. человек в I квартале 2017 года до 41,1 тыс. человек в I квартале 2018-го [6]

\section{Основные поставщики САПР}

К основным поставщикам инструментальных средств САПР на мировом рынке относятся корпорации Keysight Technologies, Synopsys, Xilinx, Cadence Design Systems, Mentor Graphics. Рынок САПР в высокой степени фрагментирован. Новые возможности поставщиков инструментальных средств САПР и производителей ИС связаны преимущественно с развитием таких секторов и технологий, как автомобильная, медицинская, промышленная электроника, ІоТ, искусственный интеллект, виртуальная/дополненная реальность. В индустрии САПР, как и в полупроводниковой промышленности в целом, продолжается процесс концентрации (сделки слияний/поглощений). Больше всего от них выигрывают крупные поставщики, предлагающие все более сложные разработки. Например, недавно корпорация Synopsys объявила об инвестициях в сфере искусственного интеллекта, который по мере масштабирования топологических норм должен помочь в разработке все более сложных конструкций ИС. Речь идет о расширении фирменной платформы разработки (Synopsys Design Platform), сокращении цикла получения результатов, достижении очередных уровней автоматизации и установлении новых стандартов качества результатов в сфере цифровых и заказных конструкций. Другой пример - компания Mentor Graphics, сформировавшая альянс c VPIphotonics (фирма-первопроходец в области моделирования устройств фотоники для создания динамических и рентабельных предложений, а также решений в области аналитики, которые позволят партнерам выйти на быстроразвивающийся рынок кремниевой фотоники (активные/пассивные/гибридные ИС и системы)) [2, 3].

\section{АКТУАЛЬНЫЕ ПРОБЛЕМЫ САПР}

Поставщики инструментальных средств САПР столкнулись с тем, что не могут больше помогать полупроводниковой промышленности решать возникающие проблемы только за счет создания более крупных и совершенных инструментальных средств САПР. Им приходится иметь дело со всем спектром технологических требований, предъявляемых клиентами. Поставщики САПР обязаны

Таблица 1. Географическая структура доходов индустрии САПР за I квартал 2018 и 2017 годов. Источник: ESD Alliance

\begin{tabular}{|c|c|c|c|c|c|c|c|}
\hline & \multicolumn{6}{|c|}{ Объем доходов, млн долл. } & \multirow{2}{*}{$\begin{array}{r}\text { При- } \\
\text { рост, } \\
\%\end{array}$} \\
\hline & $\begin{array}{c}\text { Америка } \\
\text { (оба конти- } \\
\text { нента) }\end{array}$ & $\begin{array}{r}\text { Европа, } \\
\text { Ближний } \\
\text { Восток, } \\
\text { Африка }\end{array}$ & Япония & ATP & $\begin{array}{c}\text { I кв. } \\
2018 \text { г., } \\
\text { всего }\end{array}$ & $\begin{array}{c}\text { I кв. } \\
2017 \text { г., } \\
\text { всего }\end{array}$ & \\
\hline Доходы от реализации САПР & 634,8 & 225,7 & 163,1 & 342,0 & 1365,6 & 1249,5 & 9,3 \\
\hline $\begin{array}{l}\text { Средства автоматизированного проекти- } \\
\text { рования }\end{array}$ & 328,2 & 112,2 & 79,2 & 180,2 & 699,7 & 683,6 & 2,4 \\
\hline $\begin{array}{l}\text { Средства проектирования печатных плат } \\
\text { и многокристальных модулей }\end{array}$ & 59,1 & 48,9 & 48,1 & 37,2 & 193,4 & 169,9 & 13,8 \\
\hline $\begin{array}{l}\text { Проектирование ИС на физическом уровне } \\
\text { и верификация }\end{array}$ & 247,5 & 64,6 & 35,8 & 124,7 & 472,6 & 396,0 & 19,3 \\
\hline $\begin{array}{l}\text { Доходы от разработки / реализации } \\
\text { полупроводниковых СФ-блоков }\end{array}$ & 339,2 & 84,1 & 68,3 & 339,2 & 830,7 & 790,5 & 5,1 \\
\hline Доходы от оказания услуг & 50,4 & 26,7 & 16,7 & 18,7 & 112,5 & 102,5 & 9,7 \\
\hline Доходы индустрии САПР в целом & 1024,4 & 336,4 & 248,1 & 699,9 & 2308,8 & 2142,5 & 7,8 \\
\hline
\end{tabular}




\section{АКЦИОНЕРНОЕ ОБЩЕСТВО «ЗАВОД ПОЛУПРОВОДНИКОВЫХ ПРИБОРОВ»}

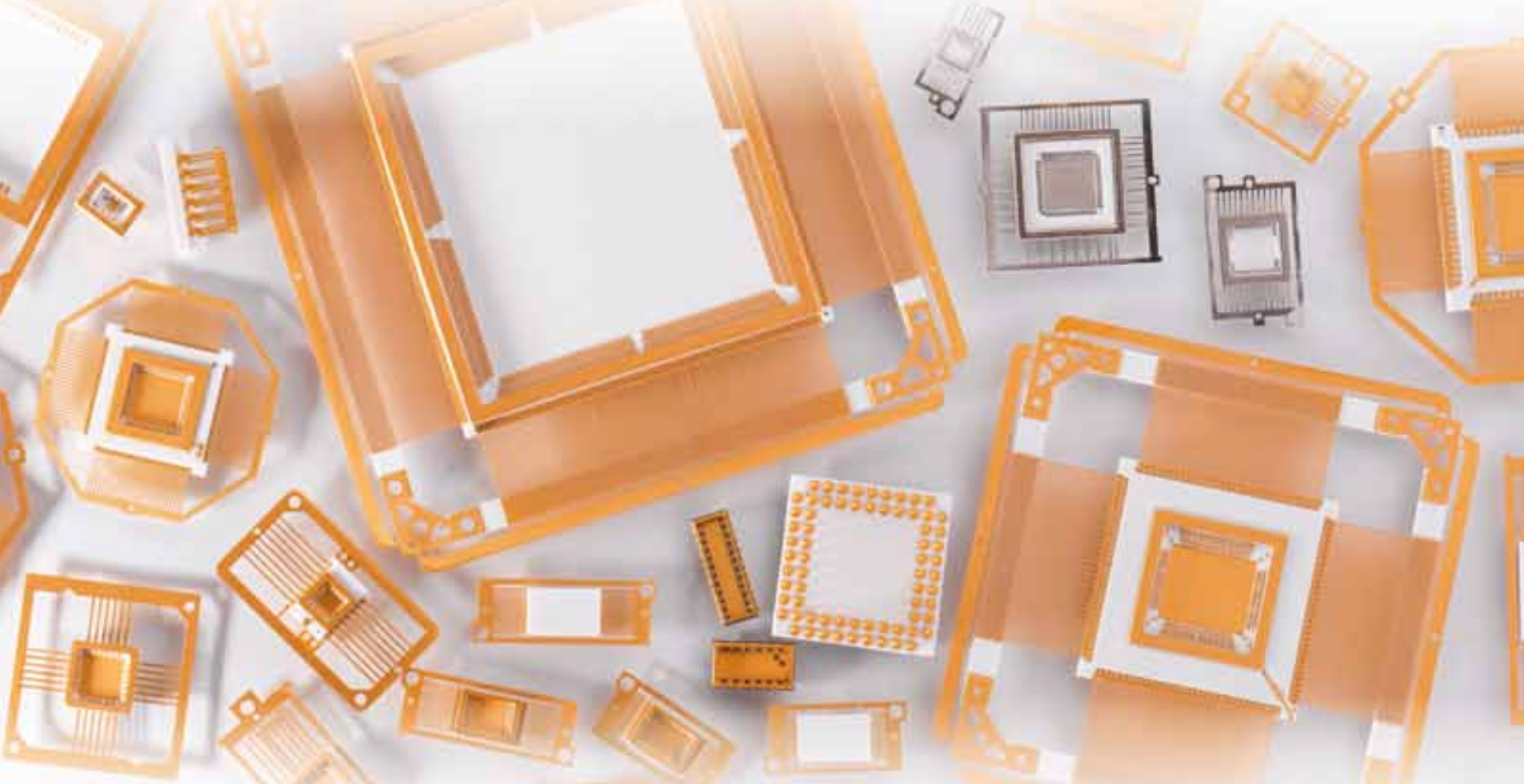

Предприятие располагает научно-технической и испытательной базой для проведения исследований, разработки и выпуска новой продукции

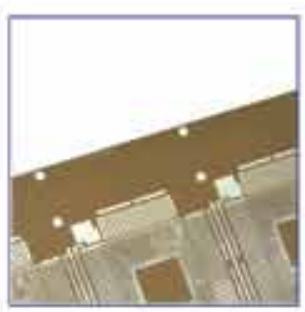

Выводные рамки

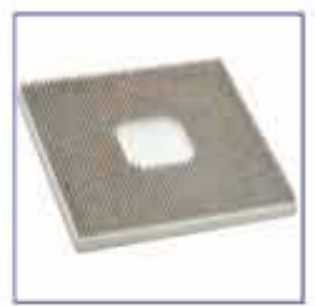

Mеталnокерамические корпуса

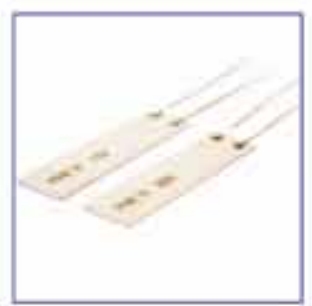

Harревательные элементы

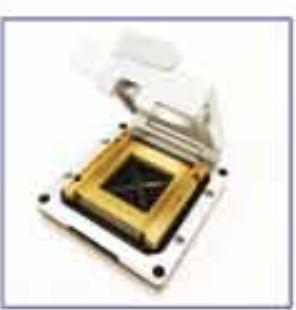

Контактные устройства

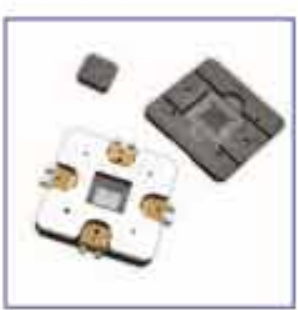

Графитовая оснастка

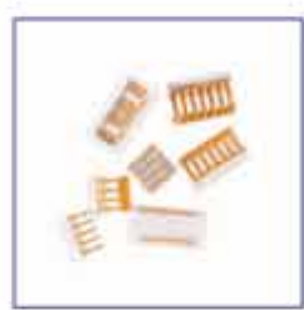

Оптозлектронные кopnyca

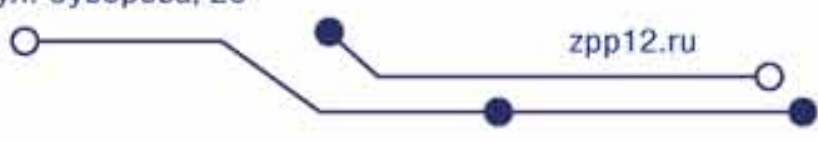


обеспечить рентабельность кремниевых технологий для заказчиков. Один из возможных подходов - предоставление клиентам дифференцированных решений и технологий, актуальных для полупроводникового бизнеса.

Современные системы-на-кристалле (CHK, SoC) могут сочетать огромное количество цифровых функций, содержать несколько процессорных ядер и аппаратных ускорителей, насчитывающих миллиарды транзисторов. Наряду с этим они могут исполнять и аналоговые функции, содержать огромные объемы встраиваемой памяти. В дополнение к аппаратной составляющей СнК отличаются огромным числом программных кодов. Освоение 3D ИС лишь обостряет проблему.

\section{Рентабельность - главное требование}

Поставщики инструментальных средств САПР обязаны обеспечить рентабельность кремниевых технологий для своих клиентов. Для этого необходимо сосредоточиться на предоставлении дифференцированных решений и технологий, учитывающих потребность в сокращении времени вывода новой продукции на рынок; наделении каждого предложения отличительными свойствами; а также требовании постоянного снижения удельных издержек при проектировании и производстве ИС; сокращении потребляемой мощности и повышении производительности. В случае реализации конструкции цифровыхприборов для создания высокопроизводительных и очень сложных решений на основе технологий с минимальными топологиями нужен полностью интегрированный поток, включающий в себя этапы от проектирования регистровых передач (RTL) до новейших систем обработки графических данных. Это обусловлено тем, что на современных технологических уровнях с минимальными топологиями для новых задач разработки и сокращения времени вывода на рынок изделий не подходят традиционные процессы проектирования. Проектировщикам нужна интегрированная методология синтеза полного кристалла, сочетающая все аспекты процесса разработки, исключающая трудоемкие и длительные неавтоматизированные этапы проектирования, уменьшающая количество связанных с кремнием (как материалом) ошибок и предотвращающая возникновение новых на завершающих этапах проектирования.

По мере увеличения размеров СнК обостряются проблемы верификации - дело не только в быстроте и точности, но и в высокой производительности, что обеспечит возможность проверки очень больших схем с точностью SРICЕ-уровня [7].

\section{ВОПРОСЫ ПРОЕКТИРОВАНИЯ ИС ПРИ МАСШТАБИРОВАНИИ}

По мере масштабирования ИС в производственный процесс вводятся новые материалы и / или предлагаются дополнительные подходы к использованию существующих, создаются перспективные конструкции, как правило, имеющие тенденцию к увеличению числа слоев. Все это учитывается в инструментальных средствах САПР. Интерес представляет исследование процессов металлизации, выполненное Национальным университетом Тайваня в 2018 году.

Важный аспект проектирования ИС с несколькими слоями металлизации - изучение влияния нижележащих металлических сплавов и потерь меди под нижней частью межслойного переходного отверстия по завершении процедур сухого травления и влажной очистки. Для уменьшения потерь меди предлагаются методы, направленные не только на увеличение выхода годных, но и на повышение надежности приборов. Все это следует учитывать при создании / модернизации инструментальных средств САПР.

По мере масштабирования размеров металлических токопроводящих дорожек пустоты в медной разводке и другие дефекты могут стать причиной отказов из-за индуцированной межслойными переходными отверстиями коррозии металлических островков. Это влияет не только на выход годных, но и на надежность приборов, в частности на миграцию электронов (electron migration, EM) и напряженности (stress migration, SM).

Для эксперимента специалисты Национального университета Тайваня выбрали процесс двойной дамасской медной металлизации, реализованный на основе подхода "сначала межслойное переходное отверстие». Медные межсоединения были сформированы в рамках завершающих операций обработки пластины (включая металлизацию - BEOL) - медного двойного дамасского процесса, который включал травление до слоя основы, осаждение диэлектрика, литографическое формирование металлических токопроводящих дорожек/ межслойных переходных отверстий и их сухое травление, жидкостную очистку после травления деионизированной водой с отработанными газами, осаждение барьерных пленок и затравочного медного слоя, электроосаждение и химико-механическую полировку меди. Формирование медной канавки поэтапно контролировалось на просвечивающем электронном микроскопе (рис. 3). Последующее осаждение барьерных пленок и процесс медной затравки лишь слегка затрагивали медную основу. Медная канавка в процессе осаждения барьерной пленки увеличивалась только на 0,3 нм. Для улучшения прилипания СuО на медную поверхность перед осаждением барьерной пленки выполнялась предварительная очистка. Важную роль в формировании медной канавки нижней части межслойного переходного отверстия сыграл процесс влажной очистки после травления.

По результатам эксперимента было установлено, что на процесс формирования и глубину медных канавок влияют сухое травление (изменение мощности плазмы), 


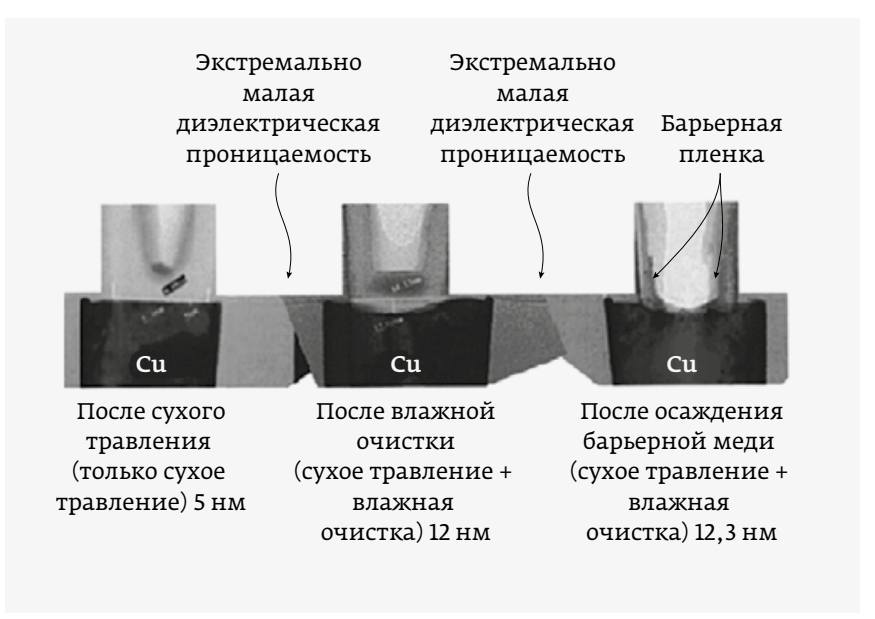

Рис. 3. Поэтапное наблюдение за формированием медной канавки на различных этапах процесса при помощи просвечивающего электронного микроскопа. Источник: Национальный университет Тайваня

влажная очистка и подача отработанного газа $\left(\mathrm{CO}_{2}\right.$, в рамках влажной очистки после сухого травления). Эти же факторы оказывают влияние и на выход годных. Так, в ходе экспериментов тайваньские ученые выявили, что нулевой рост интенсивности отказов наблюдается при снижении стандартной мощности травления на 100 Вт. При снижении мощности на 200 Вт интенсивность отказов возрастает на 10\%, повышение мощности на 100 Вт приводит к увеличению этого показателя на 6,2\%, а стандартная мощность обеспечивает рост интенсивности отказов на 1,4\%. Уменьшению интенсивности отказов способствует снижение подачи отработанного газа, основная функция которого - нейтрализация накопленного заряда, порожденного плазмой на предшествующем этапе сухого травления. Это необходимый элемент процесса влажной очистки после травления. Однако для повышения выхода годных пластин ученые Национального университета Тайваня предпочитали поддерживать режим STD-300 sccm (стандартный режим при подаче 300 стандартных кубических сантиметров в минуту) и ниже.

Таким образом, по мере масштабирования размеров металлических токопроводящих дорожек в рамках новейших методик корпусирования медная металлизация вызывает все больше опасений в плане долгосрочной надежности приборов из-за потерь меди под нижней частью межслойных переходных отверстий. Для снижения этих потерь существует ряд эффективных подходов, включая использование защищенных металлических токопроводящих дорожек, минимизацию повреждений на границе раздела за счет снижения мощности сухого травления и расхода газа на этапе очистки после травления.

все это, а также факторы, связанные с использованием при производстве ИС других материалов и структур, оказывают существенное воздействие на развитие инструментальных средств САПР по мере масштабирования ИС [8]

\section{МАШИННОЕ ОБУЧЕНИЕ И ПРОЕКТИРОВАНИЕ ИС}

Исследователи и поставщики микроэлектроники демонстрируют существенный прогресс в использовании методов машинного обучения для решения актуальных проблем проектирования современных ИС. Специалисты группы Центра разработки перспективной электроники с помощью машинного обучения (Center for Advanced Electronics through Machine Learning, (AEML) отмечают, что ранее они ориентировались в основном на целостность сигнала и мощности", а сегодня не меньшее внимание уделяется системному анализу, проектированию топологии кристалла и верифицированным методам платформенного проектирования. Они достигли значительного прогресса в области байесовских оптимизаций и сверточных нейронных сетей, относящихся к методикам DFM". Таким образом, продемонстрированы возможности встраиваемых в процесс разработки методик обучения.

Однако при разработке подобных моделей актуальна проблема доступа к фирменным данным. По словам специалистов CAEML, им удается формализовать данные из открытых источников, но их обработка занимает много времени. В целях создания продуктов свободного использования компания CAEML в 2017 году заключила соглашение с рядом поставщиков (Analog Devices, Cadence, Cisco, IBM, Nvidia, Qualcomm, Samsung и Xilinx). К открытым для обработки были отнесены данные в таких областях, как быстродействующие межсоединения, энергоснабжение, электростатический разряд системного уровня, многократно используемые СФ-ядра, проверка проектных норм.

Поставщики инструментальных средств САПР, в частности Cadence Design Systems, начали исследования в области машинного обучения в начале 1990-х годов. В 2013 году Cadence выпустила продукт Virtuoso для глубинного анализа данных применительно к извлечению сведений о паразитных явлениях в целях создания моделей машинного обучения. К настоящему времени Cadence представила более 1,1 млн моделей машинного обучения для ускорения необходимых расчетов. На следующем

\footnotetext{
Power integrity - целостность мощности, метод анализа проверки соответствия желательного напряжения силе тока от входа до выхода схемы. Один из основных показателей работоспособности электронных продуктов.

** DFM (design-for-manufacturing) - проектирование с обеспечением возможности непосредственного (прямого) внедрения (изделия) в производство (с точки зрения выхода годных, качества продукции и др.)
} 


\section{Быстрые модели}

Модели на основе машинного/непосредственного обучения позволят ускорить решение сложных вычислительных проблем и моделировать высокосложные мультивариантные нелинейные системы

\section{Примеры}

- Решения на основе методов конечных и граничных элементов (FEM/BEM) с целью решения задач извлечения данных о паразитных явлениях.

- Аналоговые макромодели ускорения вывода на рынок новых изделий (сокращение цикла вывода в 20-40 раз).

- Определение "горячих" точек конструкции с использованием методов глубокого обучения.

"Расширенный интеллект"
(системы принятия решений
на основе машинного обучения)
Знания проектировщика
"расширенного интеллекта"
в области повышения
производительности
и сокращения сроков вывода
на рынок новых изделий.
Использование накопленных
разработчиками знаний
в целях подстройки под
требования индивидуальных
клиентов, обладающих
инструментальными
средствами поставщика.
Примеры
• Рекомендации по формиро-
ванию разводки на основе
типа схемы, информации
о слоях, технологическом
уровне и наиболее важных
решениях, принятых
в последнее время.

"Расширенный интеллект" (системы принятия решении Знания проектировщика ного интеллект и сокращения сроков вывода й рынок новых изделий.

Использование накопленных требования индивидуальных

\section{Примеры} типа схемы, информации , технологическом в последнее время.

\section{Оптимизация по типу} "что, если"

Моделирование и оценка непредполагаемых или неопределенных факторов для создания надежных конструкций в интересах заказчика.

Параллельная обработка данных, используемая при решениях массовой обработки данных.

Примеры

- Модели на основе машин ного обучения и функций стоимости для оценки непредполагаемых или неопределенных данных с целью поиска оптимального и надежного создания конфигурации.

- Требуется аппаратное обеспечение с высоким параллелизмом обработки (данных).

\section{Полная машинная} автоматизация

Полная машинная автоматизация проектирования кристалла ИС, корпуса и платы на основе требований системного уровня.

\section{Примеры}

- Требования системного уровня могут зависеть от конечной конструкции. Подлежат обновлению и оптимизации.

- Допускается создание конструкций, сходных с аналогичными конструкциями или отвечающих требованиям широкого применения.

Рис. 4. Маршрутная карта корпорации Cadence Design Systems по разработке инструментальных средств САПР с использованием методов искусственного интеллекта. Источник: Cadence

этапе фирма намерена разработать средства на основе сочетания локальных и облачных систем параллельной обработки данных в целях оптимизации и ускорения процессов проектирования (рис. 4).

Специалисты TSMC планируют применять методы искусственного интеллекта при автоматизации конкретных схемных решений и технологического процесса проектирования в целом. Так, они намерены изучить возможности замены современных моделей проектирования моделями на основе искусственного интеллекта. Тем самым специалисты попытаются ускорить процесс разработки и исключить ошибки синхронизации при моделировании аналоговых ИС. Предполагается заменить подход IBIS" на этапе моделирования быстродействующих межсоединений

При создании новых схем разработчики ИС используют интеллектуальный анализ данных, статистическое обучение и другие инструментальные средства в дополнение к методам моделирования с помощью нейронных сетей, популяризируемых специалистами в области распознавания изображений (таких фирм, как Amazon, Google

\footnotetext{
IBIS (Input/output Buffer Information Specification) - специ-

фикация метода поставщиков ИС относительно предоставления

информации о буферах ввода-вывода данных потенциальным

клиентам без выявления интеллектуальной собственности их реализации и без необходимости использования собственных ключей шифрования
}

и Facebook), а также поставщиками услуг по распознаванию (идентификации) голоса.

Представители университета штата Северная Каролина сообщили об использовании суррогатных моделей на этапе окончательной оптимизации моделей физического уровня. При этом число итераций снижено до четырех по сравнению со стандартным подходом. Аналогичные методы используются при калибровке аналоговых ИС и формировании приемопередатчиков многоканальных межсоединений.

Специалисты Hewlett-Packard Enterprises (HPE) работают над использованием нейронных сетей и классификаторов гиперплоскости для прогнозирования отказов в твердотельных накопителях на основе данных о напряжении, температуре и токе.

В рамках методик машинного обучения объем обучающих данных всегда был высоким. До сих пор использовались статические классификаторы, но дальнейшее развитие требует расширения подходов. В частности, применение рекуррентных нейронных сетей позволит ввести дополнительные параметры и повысить точность обнаружения возможных системных сбоев [9].

\section{ЦЕННОСТЬ ПРИМЕНЕНИЯ ИСКУССТВЕННОГО ИНТЕЛЛЕКТА В САПР}

Что привносит искусственный интеллект в ценность систем автоматизированного проектирования (САПР)? Создание продуктов, аналогичных исходному, не приводит к формированию новой ценности. Пользователи 


\section{МЕВЛУ:АРО \\ BOEHHO МОРСКОИ САЛОН
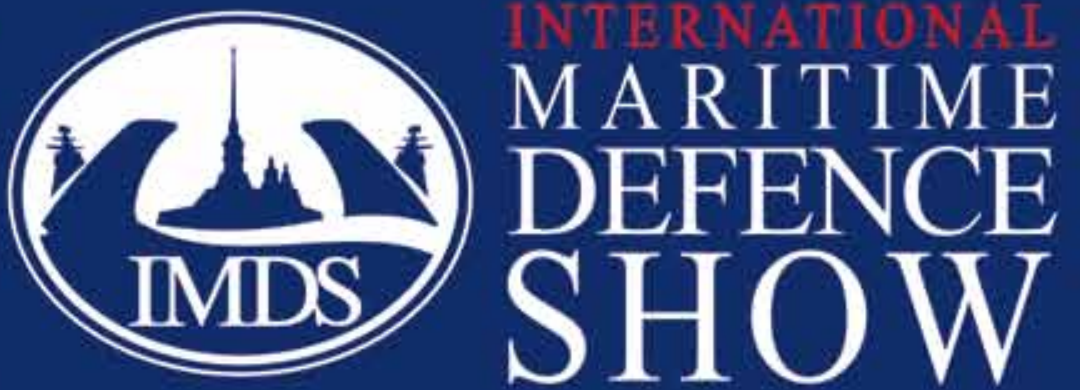

"Через сотрудничество - Кмиру и прогрессу!"

Организатор:

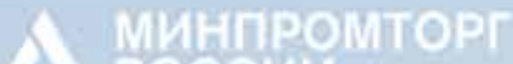

1 POCChY

При участин:
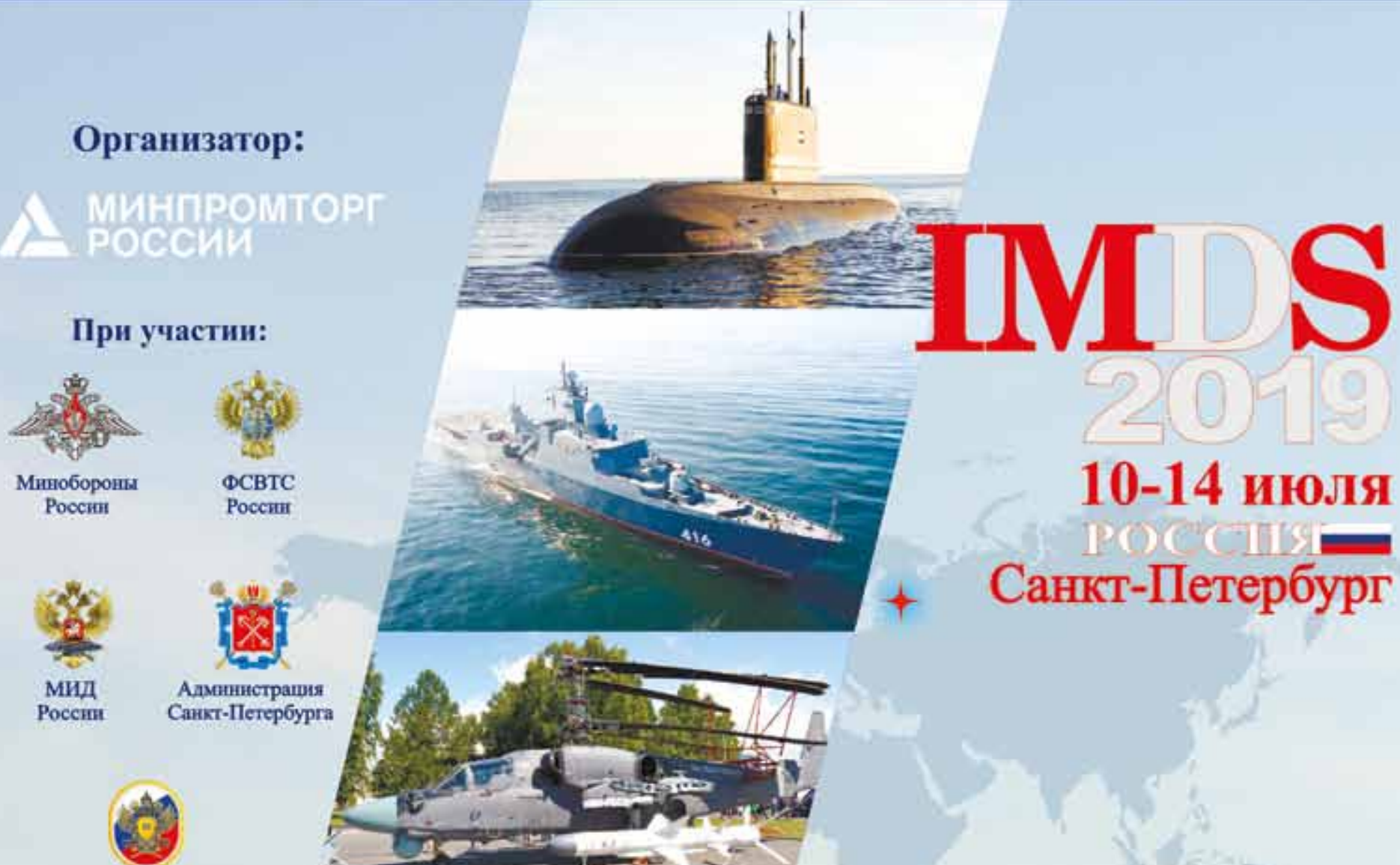

PособоРонзкспоРт

Устроитель:
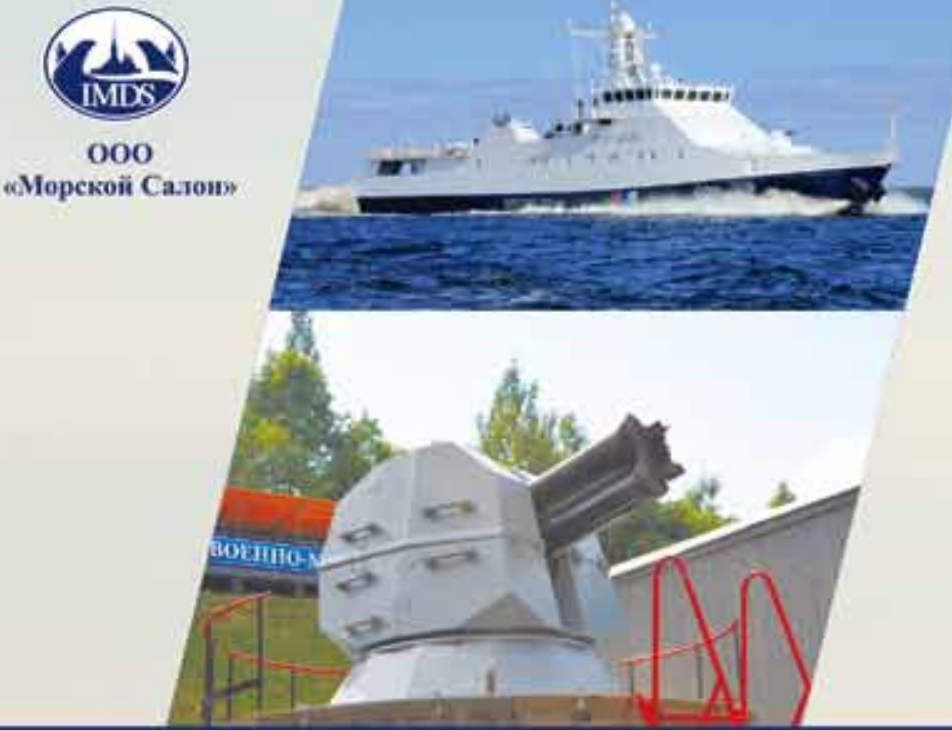

- ЭКСПОЗИЦИЯ ОБРАЗЦОВ ПРРОДУКЦИИ ПРЕДПРИЯТИЙ

- ДЕМОНСТРАЦИЯ ВООРУЖЕНИЯ И ТЕХНИКИ

- КОНФЕРЕНЦИИ, СЕМИНАРЫ, КРУГЛЫЕ СТОЛЫ, ПРЕЗЕНТАЦИИ

- VIP-ПЕРЕГОВОРЫ

- ПОСЕЩЕНИЕ ПРЕДПРИЯТИЙ 
инструментальных средств САПР, как правило, применяют ИИ для получения необходимых результатов и не занимаются улучшением базовых технологий САПР. В настоящее время разработчики инструментальных средств САПР экспериментируют с использованием ИИ ради увеличения быстродействия/производительности, появления новых функций и т. п. Основная их цель - понять реальную ценность ИИ при совершенствовании инструментальных средств САПР.

\section{Формирование базы данных}

существуют конкретные способы достижения необходимой точности. Основной метод - по важности и воздействию на конечный продукт - формирование необходимой базы данных. Машинный интеллект стал новым подходом в плане разработки инструментальных средств САПР, однако здесь таится опасность того, что неполные данные приведут к формированию неточной модели. Дело в том, что без надежных обучающих данных невозможно построить хорошие нейросетевые модели, и это может стать причиной неправильных выводов.

\section{Большая размерность}

В чем уникальность машинного интеллекта? В способности быстро решать задачи большой размерности. Проблемы инструментальных средств САПР и связаны с тем, что на протяжении многих лет их разработчики были вынуждены оперировать данными большой размерности для решения задач описания все меньших топологий. Технологии машинного интеллекта предназначены для обработки больших объемов данных, но одновременное выполнение большого числа измерений с применением методик искусственного интеллекта и глубокого обучения может привести к снижению точности измерений и даже путанице при обработке результатов. Поэтому большое внимание уделяется методам визуализации и анализа наборов данных перед их загрузкой в средства САПР.

Уменьшение размерности данных с помощью визуализации даст возможность ускорить обнаружение проблемы, а также ускорить реализацию методик машинного обучения.

\section{Технологический отбор}

Один из факторов, определяющих простоту включения машинного интеллекта в состав инструментального средства САПР, - выбор элементов разработки, то есть наборов инфраструктурных объектов, библиотек элементов и машинных языков, необходимых для разработки Ии и ПО для машинного обучения. Наиболее популярными в сфере разработки моделей глубокого обучения являются такие платформы и библиотеки, как TensorFlow, Caffe и MXNet. Но проблема состоит в том, что именно эти инструментальные средства не пользуются популярностью у разработчиков САПР, для прототипирования и создания пользовательских интерфейсов они традиционно обращаются к языкам C, C++ и Тсl. Другие разработчики предпочитают языки Python, Java, R и т. д. Кроме того, процесс развития машинного обучения подразделяется на два этапа - обучение (то есть создание самой модели) и формирование выводов (использование модели). Дополнительный вопрос - где создается модель: на сайте поставщика или заказчика? Следовательно, подгонка ИИ и встраивание процесса глубокого обучения в среду инструментальных средств САПР могут оказаться проблематичными. Ситуация осложняется тем, что инструментальные средства САПР представляют собой очень малый сегмент рынка разработки ПО, специалистов в данной области, знакомых со специфическими проблемами, мало.

Таким образом, возникает следующая ситуация:

- немногие разработчики ПО знакомы с проблемами написания программ для инструментальныхсредств автоматизированного проектирования;

- нет ответа на вопрос, как выбирать инструментарий ИИ и инструментальные средства для разработки процесса глубокого обучения;

- непонятно, как создавать интерфейсы, совместимые с инструментальными средствами разработки САПР.

При этом некоторые платформы ИИ отличаются уровнями (C/ C++), обеспечивающими точку выхода в среду для опытных разработчиков инструментальных средств САПР. Специалисты корпорации Paripath считают, что для подобных целей подходит средство TensorFlow, coпрягаемое в некоторых случаях с C/ C++.

\section{Интеграция в унаследованные" системы}

Существуют две модели усовершенствованных инструментальных средств САПР с применением ИИ. Первая использует обученную модель, предусматривающую управление процессом принятия решений в рамках соответствующего процесса в среде САПР. В этом случае обученная нейронная сеть не изменяется. Точность использования ПО не повышается в случае, если фирма-поставщик инструментального средства САПР не сохраняет базовую нейронную сеть. Подобные варианты, как правило, характерны для стандартных способов использования инструментальных средств САПР, разработанных с применением детерминированных алгоритмов.

В другом случае конечный пользователь может переобучить базовую нейронную сеть, что позволит инструментальному средству САПР со временем получать более точные результаты. Подобный вариант более

\footnotetext{
Legacy systems - унаследованные системы, системы, переставшие удовлетворять потребностям, но все еще находящиеся в эксплуатации из-за трудностей с ихзаменой.
} 


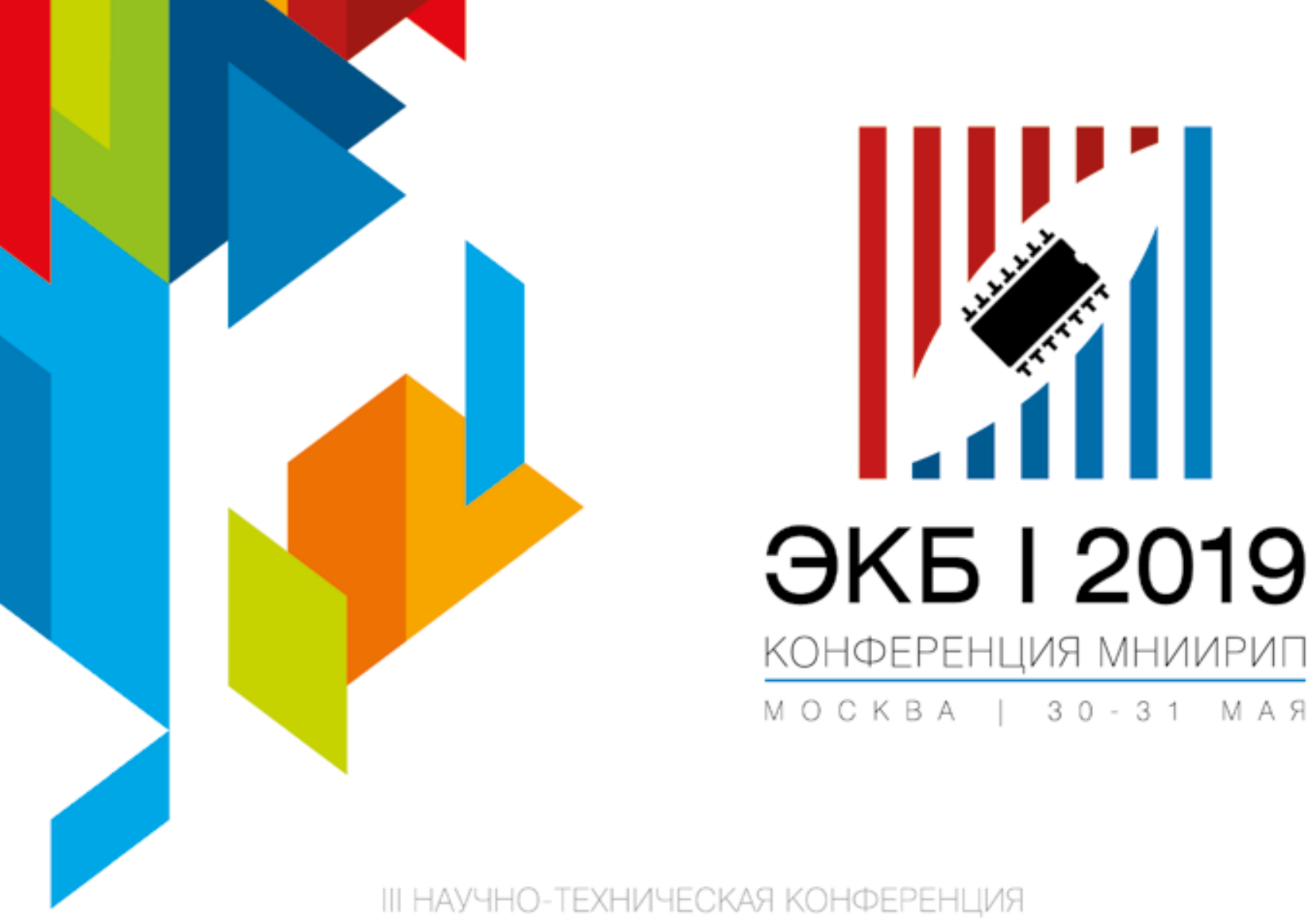

\section{«МНОГОФУНКЦИОНАЛЬНЫЙ ЦЕНТР РАДИОЭЛЕКТРОНИКИ - ЕДИНОЕ ОТРАСЛЕВОЕ ИНФОРМАЦИОННОЕ ОКНО»}

\section{ВОПРОСЫ К ОБСУЖДЕНИЮ:}

1. ДОКУМЕНТАЛЬНОЕ СОПРОВОЖДЕНИЕ РАЗРАБОТКИ И ПРОИЗВОДСТВА ЭКБ

2. ВКЛЮЧЕНИЕ ИЗДЕЛИЙ В ПЕРЕЧЕНЬ ЭКБ, РАЗРЕШЕННОЙ ДЛЯ ПРИМЕНЕНИЯ ПРИ РАЗРАБОТКЕ, МОДЕРНИЗАЦИИ, РЕМОНТЕ И ЭКСПЛУАТАЦИИ ВВСТ

3. СНЯТИЕ ИЗДЕЛИЙ ЭКБ С ПРОИЗВОДСТВА

4. ПРИМЕНЕНИЕ ЭКБ ИНОСТРАННОГО ПРОИЗВОДСТВА

5. СЕРТИФИКАЦИЯ В ОБЛАСТИ ЭКБ

6. ПРОВЕДЕНИЕ ИСПЫТАНИЙ ЭКБ И РЭА

7. СОГЛАСОВАНИЕ ПРОТОКОЛОВ РАЗРЕШЕНИЯ ПРИМЕНЕНИЯ
ЭКБ В РЕЖИМАХ И УСЛОВИЯХ, НЕ УСТАНОВЛЕННЫХ В ТЕХНИЧЕСКИХ УСЛОВИЯХ

8. ПРОВЕДЕНИЕ ОБЯЗАТЕЛЬНОЙ МЕТРОЛОГИЧЕСКОЙ ЭКСПЕРТИЗЫ

9. ПРОВЕДЕНИЕ АНАЛИЗА ОТКАЗОВ ЭКБ

10. КОНТРОЛЬ ВЫПОЛНЕНИЯ НИОКР

11. ВЕРИФИКАЦИЯ И ВАЛИДАЦИЯ МАТЕМАТИЧЕСКИХ МОДЕЛЕЙ ЗКБ

12. ОЦЕНКА ПРОГРАММНО-АППАРАТНЫХ КОМПЛЕКСОВ НА ОТЕЧЕСТВЕННОЙ ЭКБ
ДАТЫ ПРОВЕДЕНИЯ:

\section{0-31 МАЯ 2019 ГОДА}

ОРГАНИЗАТОР:

ФГУП «МНИИРИП»

ГОЛОВНАЯ НАУЧНО-ИССЛЕДОВАТЕЛЬСКАЯ ОРГАНИЗАЦИЯ МИНПРОМТОРГА РОССИИ В ОБЛАСТИ ЭКБ
ОРГАНИЗАЦИОННЫЙ КОМИТЕТ:

тел. +7 (495) 586-17-21 (доб. 416)

e-mail: gnu@mniirip.ru

ПОДРОБНОСТИ НА САЙТЕ:

WWW.mniirip.ru

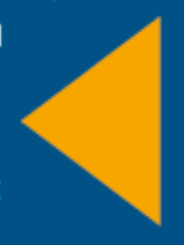


беспроигрышный, поскольку пользователи могут совершенствовать приобретенные средства без помощи профильных специалистов поставщика инструментальных средств САПР. Если переобученные модели отправляются обратно поставщику инструментальных средств САПР (для включения в новые версии решения), то преимущества от данных, полученных в процессе машинного обучения конкретного пользователя, могут стать доступными не только для поставщика первоначального средства, но и для всех пользователей этих средств.

В современной бизнес-среде инструментальных средств САПР большая часть наборов данных являются собственностью их разработчиков и тщательно охраняются. Однако логика развития инструментальных средств САПР (особенно с использованием методик машинного интеллекта) приводит к раскрытию содержания подобных наборов данных. Внедрение машинного интеллекта позволяет каждому пользователю инструментальных средств САПР расширять свои возможности благодаря обучению приобретенной продукции, но здесь многое будет зависеть от правового оформления приобретенного продукта [10].

$$
\because
$$

Развитие индустрии САПР связано не только с масштабированием ИС, но и с более интенсивным их применением на более зрелых технологиях. Действительно, Ис для Интернета вещей и ряда других перспективных секторов их конечного применения не требуют использования минимальных проектных норм и "экзотических" материалов. Вопросы обеспечения рентабельности достаточно хорошо проработаны, поэтому больший интерес вызывают перспективы применения в САПР искусственного интеллекта.

Это не только решает задачу сокращения доли "ручного" труда при проектировании, но и ускоряет вывод на рынок новых / модернизированных приборов. Другая перспектива - "персонализация" и самообучение САПР Благодаря использованию искусственного интеллекта система может запоминать наиболее удачные решения и подходы проектировщика, интегрировать их в свои библиотеки для сохранения и расширения выбора проектных решений.

Однако пока системы искусственного интеллекта относительно просты. В качестве их шаблонов часто используют мозг медоносных пчел ( 950 тыс. нейронов по сравнению со 100 млрд нейтронов учеловека), который можно рассматривать как сенсорную систему, способную интегрировать данные по типу сочетания различных датчиков, что обеспечивает принятие сложных решений [11].

Таким образом, для развития методик искусственного интеллекта, машинного и глубокого обучения, предназначенных для использования в САПР, существуют большие возможности. Как они будут реализовываться, покажет время.

\section{ЛИТЕРАТУРА}

1. Merritt R. China, Chips and 2019 Still Unclear // EE Times. 01.25.19. https://www.eetimes.com/document.asp?doc_ id $=1334240$

2. Electronic Design Automation Tools (EDA): Worldwide Market to Register a CAGR of 10.1\% during 2019-2024 // PRNewswire. March 21. 2019. https://www. prnewswire. com/news-releases/electronic-design-automationtools-eda-worldwide-market-to-register-a-cagr-of-101-during-2019-2024-300816361.html.

3. Electronic Design Automation Tools (EDA) Market - Growth, Trends, and Forecast (2019-2024). Mordor Intelligence, March 2019. https://www.mordorintelligence.com/industryreports/electronic-design-automation-eda-tools-market.

4. Cloud Electronic Design Automation (EDA) Market 2018: Historical Analysis, Developments, Emerging Technologies, Sales Revenue, Future Trends, Competitive Landscape and Opportunity Assessment Till 2022 // Market Research Future. June 2017. https://www.marketresearchfuture.com/reports/ cloud-electronic-design-automation-market-3994

5. Garrison R. 2018 Electronic Design Automation Services (EDA) Market Complete Industrial Overview Including Market Growth, Market Demand and Forecast Research Report up to 2023 // Rocket Herald News. March 2019. https://rocketherald.com/8914/2018-electronic-designautomation-services-eda-market-complete-industrialoverview-including-market-growth-market-demand-andforecast-research-report-up-to-2023/

6. Ed Sperling. EDA, IP Sales Strong Everywhere // Semiconductor Engineering. July 24 ${ }^{\text {th }}$. 2018. https:// semiengineering.com/eda-ip-sales-strong-everywhere/

7. Zahiri B. Viewpoint: EDA vendors must focus on making silicon profitable for their customers // EE Times. 06.20.11 https://www.eetimes.com/author.asp?section_ id=36\&doc_id=1285206\&utm_source=eetimes\&utm_ medium=relatedcontent

8. Cheng-Han Lee and Ren-Kae Shiue. Mechanism and improvements of Cu voids under via bottom // Solid State Technology. Wafer News. March 26. 2018. http://electroiq. com/blog/2018/03/mechanism-and-improvements-of-cuvoids-under-via-bottom/

9. Merritt R. Al Expands Role in Design // EE Times. 1.31.2018. https://www.eetimes.com/document.asp?doc_id=1332917.

10. Sharma R. How to add machine intelligence or AI to EDA tools // Solid State Technology. Wafer News. January 30. 2019. https://electroiq.com/2019/01/how-to-add-machineintelligence-or-ai-to-eda-tools/

11. Yoshida J. Al's Limits Send Scientists Back to the Brain // EE Times. 2.14.2018. https://www. eetimes.com/document. asp?doc_id=1332966. 